\title{
Microstrip Patch Antenna Array Design Anaylsis for 5G Communication Applications
}

\author{
Rovin Tiwari \\ ECE \\ Amity University \\ Gwalior, India \\ rovintiwari@gmail.com
}

\author{
Raghavendra Sharma \\ ECE \\ Amity University \\ Gwalior, India
}

\author{
Rahul Dubey \\ ECE \\ MITS \\ Gwalior, India
}

\begin{abstract}
A research on Antenna design and simulation is a emerging area among researchers. Antenna is a basic element for wireless communication. There are various shaps and types of antenna, which uses in different allpication. Now a days Microstrip patch anteena is very useful in advance electronics devices applications. This paper focused on study based various types of microstrip antenna. Return loss, VSWR, bandwidth, resonant frequency and gain is key parameters to judge antenna performance. Good value of return loss is less than -10dB. Considerable range of VSWR is 1-2. CST microwave studio is a advance software to design and simulation of all types of antenna, filter etc.
\end{abstract}

Index Terms-Antenna, Bandwidth, Return loss, CST, Microstrip, Resonant.

\section{INRODUCTION}

The Microstrip antenna was first design in 1950, but after 1970 this is most common and advance type of antenna after develop printed circuit board (PCB). During last some year microstrip antenna is most popular antenna in the telecommunication field. Due to some advantage like small in size and weight, low manufacturing cost, reliability,easy in fabrication and some drawback like low bandwidth, low gain etc. Simple diagram of microstrip patch antenna show in the fig. 1 in this fig show that microstrip antenna consist of a patch (like circular, rectangular, ellipse, ring or any other type of shape)and ground plane between theme a substrate, having particular value of dielectric constant. Dimension of ground plan and substrate plan are larger than compare to patch. Dimension of the patch . dimension of patch depend on dielectric constant of the substrate and resonance frequency of the antenna. Microstrip feed line and patch are conected by photo etched on substrate.

There are two major frequency ranges under $5 \mathrm{G}$ constraintsLower frequency- $600 \mathrm{MHz}$ to $8 \mathrm{GHz}$
Upper Frequency - $18 \mathrm{GHz}$ to $84 \mathrm{GHz}$

Due to lower frequencies, L-Band is easiest to implement for marine satellite stabilised systems. There is not much L-Band bandwidth available. The higher you go in frequency, the more bandwidth is available, but the equipment needs to be more sophisticated.

The antenna is classified based on band-

(i) Wideband

(ii) Ultra wideband

(iii) Super wideband

Another classification is based on sattlite band which is as follows-

L-Band (1-2 GHz) | S-Band (2-4 GHz) |

C-Band (4-8 GHz) $\mid$ X-Band (8-12 GHz) $\mid$

Ku-Band (12-18 GHz) |K-Band (18-27 GHz)|

K-Band (27-40 GHz)|

If one could equate the cost and availability of L-Band space segment to say, city real estate, C-Band might be the suburbs, Ku band the countryside and Ka-Band the prairies of the Wild West. Maybe a little more difficult to get to, but a lot of it available at a reasonable price.

\subsection{L-Band (1-2 GHz)}

Being a relatively low frequency, L-band is easier to process, requiring less sophisticated and less expensive RF equipment, and due to a wider beam width, the pointing accuracy of the antenna does not have to be as accurate as the higher bands. Only a small portion $(1.3-1.7 \mathrm{GHz})$ of $\mathrm{L}-\mathrm{Band}$ is allocated to satellite communications on Inmarsat. Inmarsat uses L-band for their Fleet Broadband, Inmost-B and C.

L-Band is also used for low earth orbit satellites, military satellites, and terrestrial wireless connections like GSM mobile phones. It is also used as an intermediate frequency for satellite TV where the $\mathrm{Ku}$ or $\mathrm{Ka}$ band signals are downconverted to L-Band at the antenna LNB, to make it easier to transport from the antenna to the below deck, or indoor equipment. Since there is not much bandwidth available in Lband, it is a costly commodity. 


\subsection{S-Band (2-4 GHz)}

The $\mathrm{S}$ band is a designation by the Institute of Electrical and Electronics Engineers (IEEE) for a part of the microwave band of the electromagnetic spectrum covering frequencies from 2 to 4 gigahertz $(\mathrm{GHz})$. Thus it crosses the conventional boundary between the UHF and SHF bands at $3.0 \mathrm{GHz}$.

\subsection{C-Band (4-8 GHz)}

C-band is typically used by large ships that traverse the oceans on a regular basis and require uninterrupted, dedicated, always on connectivity as they move from region to region. The shipping lines usually lease segment of satellite bandwidth that is provided to the ships on a full time basis, providing connections to the Internet, the public telephone networks, and data back-hauls to their head office. C-band is also used for terrestrial microwave links, which can present a problem when vessels come into port and interfere with critical terrestrial links. This has resulted in serious restrictions within $300 \mathrm{Km}$ of the coast, requiring terminals to be turned off when coming close to land.

\subsection{X-Band (8-12 GHz)}

The $\mathrm{X}$ band is the designation for a band of frequencies in the microwave radio region of the electromagnetic spectrum. In some cases, such as in communication engineering, the frequency range of the $\mathrm{X}$ band is rather indefinitely set at approximately 7.0-11.2 GHz.[citation needed] In radar engineering, the frequency range is specified by the Institute of Electrical and Electronics Engineers (IEEE) at 8.0-12.0 $\mathrm{GHz}$. The $\mathrm{X}$ band is used for radar, satellite communication, and wireless computer networks.

\subsection{Ku-Band (12-18 GHz)}

$\mathrm{Ku}-\mathrm{Band}$ (pronounced Kay-You) refers to the lower portion of the K-Band. The " $u$ " comes from a German term referring to "under" whereas the "a" in Ka- Band refers to "above" or the top part of K-Band. The middle portion of K-Band is a bit of a mystery. Other than a mention of K-Band radar there are few references on the Internet to it's use.

$\mathrm{Ku}$-Band is most commonly used for satellite TV and is used for most VSAT systems on yachts and ships today. There is much more bandwidth available in $\mathrm{Ku}$-Band and it is therefore less expensive that $\mathrm{C}$ or $\mathrm{L}$-band.

The main disadvantage of $\mathrm{Ku}-\mathrm{Band}$ is rain fade. The wavelength of rain drops coincides with the wavelength of $\mathrm{Ku}$-Band causing the signal to be attenuated during rain showers. This can be overcome by transmitting extra power but this of course comes with a cost as well.

The pointing accuracy of the antennas needs to be much tighter than L-Band Inmarsat terminals, due to narrower beam widths, and consequently the terminals need to be more precise and more expensive.
$\mathrm{Ku}$ band coverage is generally by regional spot beams, covering major land areas with TV reception. VSAT Vessels moving from region to region need to change satellite beams, sometimes with no coverage in between beams. Lately this process has been improved with a transatlantic beam on Telstar $11 \mathrm{n}$, and the satellite terminals and modems being programmed to automatically switch beams.

VSAT Antenna sizes typically range from the standard 1 meter, like the SeaTel 4009 , to 1.5 meters for operation in fringe areas and, more recently, as low as $60 \mathrm{~cm}$ for spread spectrum operation.

\subsection{K-Band (18-27 GHz)}

The IEEE $\mathrm{K}$ band is a portion of the radio spectrum in the microwave range of frequencies from 18 to 27 gigahertz $(\mathrm{GHz})$. The range of frequencies in the center of the $\mathrm{K}$ band between 18 and $26.5 \mathrm{GHz}$ is absorbed by water vapor in the atmosphere due to its resonance peak at $22.24 \mathrm{GHz}, 1.35 \mathrm{~cm}$.

\subsection{Ka-Band (26.5-40 GHz)}

Ka-Band (pronounced Kay-A) is an extremely high frequency requiring great pointing accuracy and sophisticated RF equipment. Like Ku-band it is susceptible to rain fade. It is commonly used for high definition satellite TV. It is also used today for terrestrial VSAT services from companies like Hughes Networks.

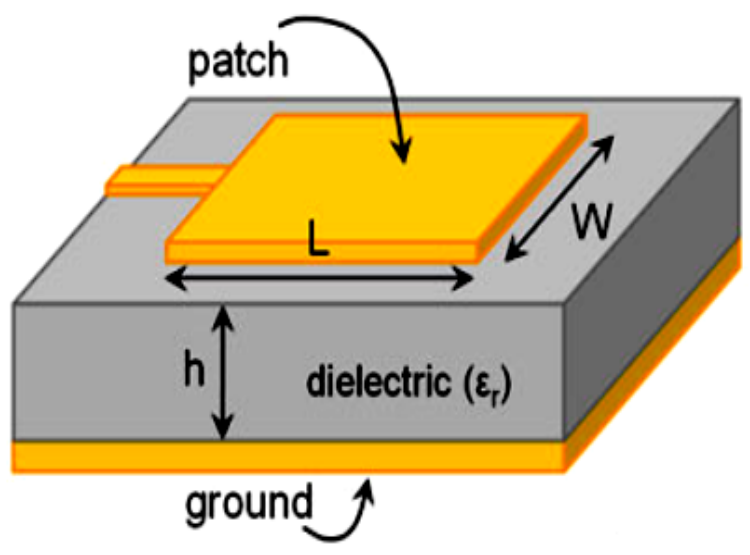

Figure 1: Microstrip patch antenna

Figure 1 shows that the basic structure of microstrip patch antenna (MPA). MPA consist 3 layes of metal amd substrate material. The bottom layer or grond structure layer is made by good conducting material or copper material. Middle layer or substrate layer is made by any dielectric material like FR4, Roggar, air etc. The top layer which is also known as patch layer, design layer is made by good conducting or copper layer material.

There are various types of microstrip patch antenna design in current scanerio for $5 \mathrm{G}$ and other applications.

Some of the efficient design name is following-

(i) Microstrip patch antenna 
(ii) Microstrip dipole antenna

(iii) Microstrip patch antenna array

(iv) Microstrip patch MIMO antenna

(v) Microstrip Fractral antenna

(vi) Substrate intregrated waveguided antenna

\section{LITERATURE SURVEY}

A. Yadav, et al.,[1] This work displays a structure of $2 \times 1$ microstrip patch array antenna for $5 \mathrm{G} \mathrm{C}$-band passage applications. The array utilizes the component of rectangular microstrip antenna with $\mathrm{U}$ opening on parasitic patch for $5 \mathrm{G} \mathrm{C}$ Band $(3.4-3.8 \mathrm{GHz})$ application. A microstrip feed arrange is utilized to bolster the antenna array is likewise actualized in the plan. The antenna is two layered antenna array and low profile is a decent up-and-comer of antenna for $5 \mathrm{G} \mathrm{C}$ band passageway applications. the paper shows consequences of examination, for example, return misfortune, efficiencies, radiation design, and so forth of both single component and array antenna.

M. Patriotis et al., [2] This work presents a broadband right hand circularly captivated (RHCP) 16-component antenna array working in the recurrence band of $20-32 \mathrm{GHz}$. The array components are shortened patches encouraged utilizing a successive pivot power divider (SRPD). The antenna can be utilized at the same time in the getting mode $(\mathrm{Rx})$ and transmitting mode (Tx) by choosing the implanted reconfigurable channels. A PIN diode reconfigurable bandpass channel (BPF) is utilized at the Tx port so as to choose the band of activity.

A. M. Yusuf, et al., [3] Unmanned Aeronautical Vehicle (UAV) is one of the stages which can bolster Manufactured Gap Radar (SAR) to distinguish an objective in $\mathrm{C}$ and $\mathrm{X}$ band. The innovation is generally modest and can be worked in any climate condition. In any case, constrained capacity of UAV for conveying payload drives specialist to construct SAR gadget as little and light as conceivable including the sensor, in this term is the antenna. In this examination, a double band microstrip antenna array $1 \times 8$ at $\mathrm{C}$-band $(5.8 \mathrm{GHz})$ and $\mathrm{X}$ band $(9.65 \mathrm{GHz})$ has been planned and fabricated on FR-4 substrate. E-Formed patch has been actualized in this antenna to accomplish double reaction recurrence.

N. Yan, et al., [4] A tale stacked-patch antenna dependent on substrate-integrated suspended line innovation is proposed. The antenna is intended to act naturally bundled utilizing five substrate layers with installed air holes. By means of is used to associate the antenna driven patch with the feed line, and afterward resounding recurrence at $5.2 \mathrm{GHz}$ is produced. Uformed opening is scratched on the antenna driven patch for impedance coordinating. So as to additionally expand the impedance bandwidth, a stacked patch with incline space is presented and afterward another resounding recurrence at 6.2 $\mathrm{GHz}$ is created.

V. Ingle et al.,[5] Channel estimation plays affective role on the performance of wireless and wire communication systems, since its knowledge is utilized to track and analysis the signal data symbols. Channel estimation is very important technique especially in wireless network systems where the wireless channel change over time, usually caused by transmitter and/or receiver being in motion and/or stable. In order to provide stability and high data receiving rates at the receiver, the system needs an accurate and minimum error estimate of the time-varying channel.

M. Alibakhshikenari et al., [6] The fractals are made out of four interconnected-'Y-formed' openings that are isolated with a modified 'T-molded' space. The MTM-EMBG structure is set between the individual patch antennas in a $2 \times 2$ antenna exhibit. Estimated results show the normal between component detachment improvement in the recurrence band of intrigue is 17,37 and $17 \mathrm{~dB}$ between radiation components \#1 and $\# 2, \# 1$ and $\# 3$, and \#1 and \#4, separately. With the proposed strategy there is no requirement for utilizing metallicby means of gaps.

X. Yang, et al., [7] A minimal patch antenna exhibit with high segregation by utilizing two decoupling structures including a column of fractal uniplanar conservative electromagnetic bandgap (UC-EBG) structure and three cross openings is proposed. Reenacted results show that critical improvement in interelement disconnection of $13 \mathrm{~dB}$ is acquired by setting the proposed fractal UC-EBG structure between the two emanating patches. Additionally, three cross spaces carved on the ground plane are acquainted with further stifle the common coupling. The plan is anything but difficult to be produced without the execution of metal vias, and a progressively minimized cluster with the edge-to-edge separation of $0.22 \lambda 0$ can be encouraged by a line of fractal UC-EBG, which can be very much applied in the patch antenna exhibit.

P. R. Prajapati et al., [8] To improve the exhibition parameters of patch antenna, for example, hub proportion (AR) bandwidth, return misfortune bandwidth, radiation productivity, etc another strategy of blend of fractal hypothesis and surrendered ground structure (DGS) is proposed without precedent for plan of CP antennas. 44.74\% size decrease in patch size, improvements of $62.73 \%$ in AR bandwidth, $70.74 \%$ consequently misfortune bandwidth and $4.03 \%$ in radiation effectiveness is accomplished as contrasted and ordinary patch antenna, after joining of Koch bend fractal DGS in the ground plane.

S. Costanzo et al., [9] A Minkowski fractal-molded patch is proposed for the plan of a decreased size reflectarray component. The inherent scaling down capacity of the fractal geometry is completely abused by leaving unaltered the patch length, while utilizing the fractal scaling element to get a decent reflectarray stage dexterity. The accomplished size decrease impact permits to pick cluster lattices with littler interelement separating, in this way offering the chance to have wide-point filtering abilities. 
S.Pandit, et al.,[10] This letter displays a novel low-profile high-gain antenna with cross-polarization (x-pol) concealment utilizing cross circular loop resonator (CCLR) metamaterial (MTM) slab in substrate-integrated waveguide-bolstered space antenna (SIW-SA). The SIW-SA antenna, which is the reference antenna, works at $9.73 \mathrm{GHz}$. The CCLR MTM slab goes about as a low-impedance slab, which is put in the superstrate of the reference antenna at the stature of just $\lambda 0 / 10$, where $\lambda 0$ is the free-space wavelength at the reverberation recurrence of the antenna.

S. Adhikari, et al.,[11] Estimated periods of forward and turn around transmission coefficients show the non-proportional conduct of this transmission line. Estimated results outlining the examination between a solitary ferrite slab-stacked SIW and a HMSIW are exhibited. As an application model, a twodimensionally tunable band pass channel (BPF) working at $\mathrm{X}$-band is proposed and contemplated. By controlling the estimation of stacked lumped capacitors and the applied attractive inclination, a bandwidth tunable BPF is illustrated.

X. Zou,et al.,[12] A dielectric stacked antipodal curvilinear decreased opening antenna dependent on substrate integrated waveguide is proposed in this investigation. High gain, low side flap level and low reflectance could be acquired by picking of the length and width of space accurately. The stacked dielectric slab before the opening can bring about a smaller beamwidth both in the $\mathrm{H}$ - plane and in the $\mathrm{E}$ - plane as a dielectric directing structure. A two-dimensional antenna exhibit, framed by a non-planar four-way power divider, has a wide bandwidth running from 9.5 to $12 \mathrm{GHz}$ and high gains more than $12 \mathrm{dBi}$, which can be widely applied to microwave and millimeter-wave frameworks.

\section{Challenges AND APPLiCATIONS}

\subsection{Designing Calculations}

One of the significant piece of antenna planning is the choice of substrate which has specific dielectric consistent and ought not change its qualities in any conditions.

\subsection{Simulation Process}

Indeed, even a little change in measurements of patch influences the bordering fields from the edges. It influences the powerful length, along these lines changing the reverberation recurrence. In the recreation procedure doling out of waveport is significant. The feed is nourished with coaxial link with appropriate adjustment of antenna with short out and open circuit present and legitimate end of transmission line while there is no such idea of encouraging through link present in the CST designing. Along these lines, the vitality is furnished with the assistance of a sheet called as waveport, put toward the start of the feedline to give excitation to the waveport. Doling out legitimate limit conditions in reproduction process is most basic parameter. A limit can be doled out to any two-dimensional zone, for example, a plane, a face of an article or an interface between two items. Most limit conditions are utilized to characterize electromagnetic attributes, for example, conductivity or resistivity. This additionally incorporates energizing the structure, and henceforth any mistake can bring about mistaken outcomes.

\subsection{Fabrication and Testing Process}

There is a little variety in the parameters considered in recreation procedure and results got after manufacture process. After creation of antenna, antenna transmits in the air. At the hour of radiation of antenna, there are numerous metallic articles present in nature which influences the engendering of electromagnetic waves. Because of these articles, impressions of EM waves occur. This prompts the variety in radiation example of antenna. Along these lines, we get variety in the antenna qualities. The distinctions in the outcomes after creation can likewise have an explanation of assembling deserts. It might contain contaminations present in the material utilized for antenna manufacture. Likewise the ecological conditions like dampness; high temperature influences the charge dispersion of patch which influences the qualities of an antenna.

\subsection{Application}

Wi-Fi/Wi-max- Very high speed data access from internet and setup WLAN.

Automobiles- Driverless cars and other automobiles are going to rule the future.

Healthcare- Remote patient monitoring will become more efficient with IOT.

Smart Retail- Key applications of IOT in retail industry, it includes supply chain and smart store applications.

Smart home- IoT devices are a part of the larger concept of home automation, which can include lighting, heating and air conditioning, media and security systems.

Satellite communications band- For both uplink and downlink communication.

Table 1: Presented Antenna Dimensions

\begin{tabular}{|c|c|c|}
\hline PARAMETERS & DESCRIPTION & SIZE \\
\hline $\mathrm{L}$ & $\begin{array}{c}\text { Length of sub- } \\
\text { strate }\end{array}$ & $10 \mathrm{~mm}$ \\
\hline $\mathrm{W}$ & Width of substrate & $10 \mathrm{~mm}$ \\
\hline $\mathrm{Lf}$ & $\begin{array}{c}\text { Length of feed } \\
\text { line }\end{array}$ & $4 \mathrm{~mm}$ \\
\hline $\mathrm{Wf}$ & Width of feed line & $1 \mathrm{~mm}$ \\
\hline $\mathrm{A}$ & $\begin{array}{c}\text { Major axis of el- } \\
\text { liptical slot }\end{array}$ & $4.150 \mathrm{~mm}$ \\
\hline $\mathrm{B}$ & $\begin{array}{c}\text { Minor axis of el- } \\
\text { liptical slot }\end{array}$ & $2.075 \mathrm{~mm}$ \\
\hline $\mathrm{R}$ & $\begin{array}{c}\text { Radius of sector } \\
\text { patch }\end{array}$ & $1.5 \mathrm{~mm}$ \\
\hline
\end{tabular}

In any case, intrinsically MPA have thin data transfer capacity so to upgrade transmission capacity different procedures are 
locked in. Today Specialized gadgets bolster a few applications which require higher data transmission, for example, cell phones nowadays are getting more slender and more brilliant yet numerous application upheld by them require higher transfer speed, so microstrip antenna utilized for playing out this task ought to give more extensive transmission capacity and their size ought to be conservative with the goal that it ought to involve less space while keeping the span of gadget as little as could be expected under the circumstances. In this paper a survey of various methods utilized for conservative and broadband microstrip fix reception apparatus is given.

\section{Conclusion}

Various microstrip antenna geometry and techniques make antenna efficient for wireless band communication. Although various shape is very challenging to design and simulate due to its complexity, but there are lot of wireless application. This paper reviews of previous research work and comparative study. CST software is used to design such type of antenna. Bandwidth, return loss, resonant frequency, VSWR, gain, efficiency is key parameters to analysis of antenna design.

\section{REFERENCES}

1. A. Yadav, M. K. Saraswat, V. Palukuru and R. Gautam, "Antenna array for 5G C-band for mobile terminals," 2019 TEQIP III Sponsored International Conference on Microwave Integrated Circuits, Photonics and Wireless Networks (IMICPW), Tiruchirappalli, India, 2019, pp. 293-297.

2. M. Patriotis, F. N. Ayoub, C. G. Christodoulou and S. Jayaweera, "A K/Ka Band Frequency Reconfigurable Transmit/Receive Antenna Array," 2019 13th European Conference on Antennas and Propagation (EuCAP), Krakow, Poland, 2019, pp. $1-4$

3. A. M. Yusuf, H. Wijanto and Edwar, "Dual C-X-Band EShaped Microstrip Antenna Array $1 \times 8$ for Synthetic Aperture Radar on UAV," 2019 IEEE International Conference on Signals and Systems (ICSigSys), Bandung, Indonesia, 2019, pp. 186-189.

4. N. Yan, K. Ma and H. Zhang, "A Novel Substrate-Integrated Suspended Line Stacked-Patch Antenna Array for WLAN," in IEEE Transactions on Antennas and Propagation, vol. 66, no. 7, pp. 3491-3499, July 2018

5. V. Ingle and A. Patel, "Adaptive Channel Estimation Algorithm For Multi Input Multi Output System - A Brief Review”, SMART MOVES JOURNAL IJOSCIENCE, vol. 2, no. 1, Feb. 2016. https://doi.org/10.24113/ijoscience.v2i1.69.

6. M. Alibakhshikenari et al., "Study on isolation improvement between closely-packed patch antenna arrays based on fractal metamaterial electromagnetic bandgap structures," in IET Microwaves, Antennas \& Propagation, vol. 12, no. 14, pp. 2241 2247, 28112018.

7. X. Yang, Y. Liu, Y. Xu and S. Gong, "Isolation Enhancement in Patch Antenna Array With Fractal UC-EBG Structure and
Cross Slot," in IEEE Antennas and Wireless Propagation Letters, vol. 16, pp. 2175-2178, 2017.

8. P. R. Prajapati, G. G. K. Murthy, A. Patnaik and M. V. Kartikeyan, "Design and testing of a compact circularly polarised microstrip antenna with fractal defected ground structure for L-band applications," in IET Microwaves, Antennas \& Propagation, vol. 9, no. 11, pp. 1179-1185, 2082015 .

9. S. Costanzo and F. Venneri, "Miniaturized Fractal Reflectarray Element Using Fixed-Size Patch," in IEEE Antennas and Wireless Propagation Letters, vol. 13, pp. 14371440, 2014.

10. S. Pandit, A. Mohan and P. Ray, "A Low-Profile HighGain Substrate-Integrated Waveguide-Slot Antenna With Suppressed Cross Polarization Using Metamaterial," in IEEE Antennas and Wireless Propagation Letters, vol. 16, pp. 1614-1617, 2017.

11. S. Adhikari, S. Hemour, A. Ghiotto and K. Wu, "Magnetically Tunable Ferrite-Loaded Half-Mode Substrate Integrated Waveguide," in IEEE Microwave and Wireless Components Letters, vol. 25, no. 3, pp. 172-174, March 2015.

12. X. Zou, C. Tong, J. Bao, J. Sun and C. Li, "Dielectric loaded antipodal curvilinear tapered slot antenna based on substrate integrated waveguide," in IET Microwaves, Antennas \& Propagation, vol. 8, no. 13, pp. 1113-1119, 21 October 2014.

13. H. Oraizi and S. Hedayati, "Miniaturization of Microstrip Antennas by the Novel Application of the Giuseppe Peano Fractal Geometries," in IEEE Transactions on Antennas and Propagation, vol. 60, no. 8, pp. 3559-3567, Aug. 2012.

14. J. Eichler, P. Hazdra, M. Capek, T. Korinek and P. Hamouz, "Design of a Dual-Band Orthogonally Polarized L-Probe-Fed Fractal Patch Antenna Using Modal Methods," in IEEE Antennas and Wireless Propagation Letters, vol. 10, pp. 1389-1392, 2011.

15. J. J. Casanova, J. A. Taylor and J. Lin, "Design of a 3-D Fractal Heatsink Antenna," in IEEE Antennas and Wireless Propagation Letters, vol. 9, pp. 1061-1064, 2010. 